\title{
Modification Goal Programming for Solving Multi-Objective De Novo Programming Problems
}

\author{
Febrianto Afli*, Ihda Hasbiyati, Moh Danil Hendry Gamal \\ Department of Mathematics, University of Riau, Pekanbaru, Indonesia \\ Email address: \\ Febrianto.afli17@gmail.com (F. Afli), ihdahasbiyati@gmail.com (I. Hasbiyati),mdhgamal@unri.ac.id (M. D. H. Gamal) \\ ${ }^{*}$ Corresponding author
}

To cite this article:

Febrianto Afli, Ihda Hasbiyati, Moh Danil Hendry Gamal. Modification Goal Programming for Solving Multi-Objective De Novo Programming Problems. International Journal of Management and Fuzzy Systems. Vol. 5, No. 4, 2019, pp. 64-69.

doi: $10.11648 /$ j.ijmfs.20190504.11

Received: October 25, 2019; Accepted: November 15, 2019; Published: November 21, 2019

\begin{abstract}
Many methods can be used to solve multi-objective problems, but not all of them provide truly optimal results because there are still deviations and inefficient use of resources so that they still produce residuals. Resources that are not used in their entirety can reduce the level of optimization in solving multi- objective problems. This happens because we are too forced to solve existing problems rather than redesigning the problem so that it gets satisfactory results. One method that can be used to solve this problem is by using the de novo program. The de novo programming aims to design a more optimal system by expanding resources based on available budgets. The de novo programming changes the function of constraints into form of a budget. This change into one constraint function makes in the feasible solution changes. So it is important to determine the goal for all objectives that have the same importance so that all objectives are achieved at the optimum condition. The objectives of the goals to be achieved must be determined in advance in resolving multi-objective problems. This paper proposes determining the goal objectives using the average concept for objectives that have the same interests. Determination of goals with an averageeachconcept considers the objectives of other goals in determining a goal. Determination of goal objectives using the average concept applied to the goal programming to solve the multi-objective problem of the de novo programming. Solution to the de novo program's multi-objective problem using a modified goal program. The computational results with benchmarking problems show that the proposed method gives satisfactory results and more practical work.
\end{abstract}

Keywords: De Novo Programming, Multi-Objective Optimization, Goal Programming

\section{Introduction}

One method that can be used in multi-objective problem decision making is the one using the de novo programming. Zeleny argues that the de novo programming is a way to see a system where in addition to optimizing existing systems, it also suggests planning an optimal system based on the objective function to obtain high productivity [12].

The de novo programming approach resolves optimization problems with a total approach system, meaning that in addition to determining the best combination of outputs, it also provides an integrated proposal for the use of raw materials through the available budget. In the de novo programming method the constraints of raw materials will be arranged as efficiently as possible so as not to produce residuals.

In solving multi-objective problems, the goals for each goal to be achieved must be determined in advance by considering other objectives so that each goal is achieved in optimal conditions. Determining goals without considering other goals will make other goals not optimal. The decision variables obtained also depend on the goals of each goal. Determining different goals will provide different decision variables. Therefore, the goal objectives achieved have an important role in resolving multi-objective problems.

In solving the multi-objective problem of the de novo programming, Zeleny uses the maximum ideal solution as the initial solution to get the goals to be achieved from each goal [13]. After the maximum ideal solution of all objectives is 
obtained, then he calculates how much the cost or budget needed to realize all the objective functions in the maximum ideal solution or the maximum budget. Furthermore, the ratio between available budgets is soughtwith a maximum total budget. This ratio is obtained by dividing the available budget against the maximum budget. After the value of the ratio is obtained, the next stepis to determine the goals to be achieved for each goal by multiplying the ideal maximum solution with a value ratio for each destination. The result of this ideal maximum solution to the ratio becomestarget to be achieved.

Li and Lee introduced a fuzzy approach to solving de novo programming problems in general. Theyare use the maximum ideal solution value and the minimum ideal solution for each goal to set goals for each goal[7]. Determination of the objective of using fuzzy suggestions by subtracting the target to be achieved with the minimum ideal solution and dividing it by the difference between the minimum and ideal ideal solutions, where the results are small at $\lambda$, for $\lambda \in[0,1]$.

Umarusmanuses the min-max goal programming approach to solve the de novo programming multi-objective problem. Determination of the objective of the research is the value of the maximum ideal solution for each goal [11]. Umarusman made modifications to the min-max goal programming in solving the de novo programming problem. Modifications made are to add new constraints as much as the objective function to be achieved, where the constraints added are the division of the deviation variables divided by the maximum and minimum ideal solution differences whose results are smaller than the total deviation of all goals.

Banik and Bhattacarya apply a weighted goal programming approach to solve the de novo programming multi-objective problem [2]. Determination of the objectives achieved in this study is also the value of the maximum ideal solution for each goal. Theyare use normalization techniques to solve the multi-objective problems of the de novo programming that are solved using a boot goal programming. The normalization technique used is the Luhandjula technique.

Other studies using de novo programs include; Z. Babic and I. Pavic, his research Multicriterial production programming by the de novo approach program [1]. Y. M. Zhang et. all, his research of the Inexact de novo program for water resource systems planing [14]. Z. Y. Zuang and A. Hocine, his research meta goal programming approach for solving multi-objective programs de novo problems [15].

Based on the description above, it can be said that many methods can be used to solve the multi-objective problem of the de novo programming. The difference in the methods used will gives different results, both the goal and the money decision variable obtained.

In this article the authorsare interested in designing a new method for solving the multi-objective problem of the de novo programming. This study aims to determine more accurate goals of each goal so that each goal is achieved optimally. Determination of the target value of each goal in this study is to consider other decision variables which are also optimal solutions of other objective functions. Completion of the de novo multi-objective problem in this study uses a goal programming. The target function of the objective constraints on the goal programming will be modified to solve the multi-objective problem of the de novo programming. This modification is expected to solve the multi-objective problem of the de novo programming and provide a satisfying solution for all objectives to be achieved.

\section{Multi-Objective of the De Novo Programming Problems}

The de novo programming is a general multi-objective programming problem whose constraints function is arranged in the form of costs. The intended fee is the unit cost of each constraint or resource limit. So that later it can be known how much the total cost needed to solve these multi-objective problems. The existence of per unit costs from each constraint boundary is a requirement to be able to reconstruct multi-objective problems into a de novo multi-objective programming. The general form of multi-objective problems is as follows:

$$
\begin{gathered}
\max _{k}=c_{k 1} x_{1}+c_{k 2} x_{2}+\cdots+c_{k n} x_{n}, k=1,2, \ldots, r \\
\text { s.t, } \\
a_{i 1} x_{1}+a_{i 2} x_{2}+\cdots+a_{i n} x_{n} \leq b_{i}, i=1,2, \ldots, m \\
x_{j} \geq 0, j=1,2, \ldots, n
\end{gathered}
$$

with $z_{k}$ is the maximized objective function, $r$ the number of maximum objective functions, $x_{n}$ is the decision variable, and $b_{i}$ the constraint function limit or resource.

Suppose that $p_{i}$ is the unit cost of resources for $-i$ which has been given. Then rebuild into the form of a budget by multiplying the unit cost of the constraint function, as follows:

$$
p_{i}\left(a_{i 1} x_{1}+a_{i 2} x_{2}+\cdots+a_{i n} x_{n} \leq b_{i}\right), i=1,2, \ldots, m
$$

Then the coefficients having the same variable are added, so that it is obtained

$$
\begin{aligned}
& \left(p_{1} a_{11}+p_{2} a_{i 2}+\cdots+p_{m} a_{m 1}\right) x_{1} \\
& \quad+\left(p_{1} a_{12}+p_{2} a_{22}+\cdots+p_{m} a_{m 2}\right) x_{2}+\cdots \\
& +\left(p_{1} a_{1 n}+p_{2} a_{2 n}+\cdots+p_{m} a_{m n}\right) x_{n} \\
& \quad \leq \sum_{i=1}^{m} p_{i} b_{i}, i=1,2, \ldots, m
\end{aligned}
$$

Suppose that $v_{n}$ is the amount of coefficient from $x_{n}$ and $B$ is the total available budget, i.e. $B=\sum_{i=1}^{m} p_{i} b_{i}, i=$ $1,2, \ldots, m$. Inequality (2) becomes:

$$
v_{1} x_{1}+v_{2} x_{2}+\cdots+v_{n} x_{n} \leq B
$$

so, the de novo programming formulation becomes: 


$$
\begin{gathered}
\max _{k}=c_{k 1} x_{1}+c_{k 2} x_{2}+\cdots+c_{k n} x_{n}, k=1,2, \ldots, r \\
\text { s.t. } \\
v_{1} x_{1}+v_{2} x_{2}+\cdots+v_{n} x_{n} \leq B \\
x_{j} \geq 0, j=1,2, \ldots, n
\end{gathered}
$$

with $z_{k}$ is the maximum objective function, $r$ the maximum number of objective functions, $x_{n}$ is the decision variable, $v_{n}$ the cost needed to produce for- $n$ unit, and $b_{i}$ the constraint function or resource limit.

Multi-objective problem is a problem that consists of several objectives to be achieved at a predetermined level of goals. The goal is the target to be achieved in a goal. The goal of the goal is also one of the requirements to solve multi-objective problems. Determination of the goals to be achieved in resolving multiobjective problems is important, because it will affect the optimal solution obtained. The objective of the multi-objective problem is the boundary constraints of each goal. Determining different objectives will produce different decision variables. Determination of goal goals that are too large will result in other goals being achieved in less optimum conditions. Because of that, the goal setting should be determined based on the interests of all objectives so that the results obtained are the optimum results for all objectives.

The de novo programming changes the constraints of multiobjective problems into the form of a budget, so that the constraints which initially consist of several constraints then become one or single constraint. This causes a change in the function of the constraints so that the feasible area of the constraints changes. Therefore, determining the goal after the constraint function is changed in the form of the de novo programming.

The de novo programming was first introduced by Zeleny.He introduced a way to solve the de novo multiobjective problem, namely by calculating the maximum ideal solution for each objective against the constraint. Suppose $\max z_{k}=z_{k}^{*}$. Then he calculates the minimum cost to realize the goal in the maximum ideal state, namely:

$$
\begin{gathered}
\min v_{1} x_{1}+v_{2} x_{2}+\cdots+v_{n} x_{n} \\
\text { s.t. }, \\
c_{k 1} x_{1}+c_{k 2} x_{2}+\cdots+c_{k n} x_{n}=z_{k}^{*} \\
x_{j} \geq 0, j=1,2, \ldots, n
\end{gathered}
$$

with $z_{k}^{*}$ is the ideal maximum solution for each goal for- $k$.

Problem solving (4) will provide the minimum budget needed to realize each goal at the maximum ideal condition. Let $\min v_{1} x_{1}+v_{2} x_{2}+\cdots+v_{n} x_{n}=B^{*}$. Then he calculates the ratio between the available budget for the minimum budget needed to realize the goal in the maximum ideal condition, as follows:

$$
r=\frac{B}{B^{*}}
$$

Furthermore, this ratio is uses to determine the goals to be achieved for each goal by multiplying the maximum ideal solution for each destination. The formula for solving multiobjective problems introduced by Zeleny can be written as follows:

$$
\begin{gathered}
c_{k 1} x_{1}+c_{k 2} x_{2}+\cdots+c_{k n} x_{n}=z_{k}^{*} r, k=1,2, \ldots, r \\
\text { s.t. } \\
v_{1} x_{1}+v_{2} x_{2}+\cdots+v_{n} x_{n} \leq B \\
x_{j} \geq 0, j=1,2, \ldots, n
\end{gathered}
$$

with $z_{k}^{*} r$ is the goal to be achieved for each goal.

\section{Determination of Goal Objectives}

In this study, determining the target goals for each goal taking into account the decision variables of the ideal solution for other goals. This means that the goal to be obtained is not only the maximum ideal solution for that objective, but that goal is the goal to be achieved by considering the interests of all objectives. Decision variables from the ideal solution to other goals will be taken into account to determine the goal of each goal. The ideal maximum solution is the maximum solution of a goal when it is resolved against constraints without including other objectives or solutions to a goal when calculated in linear programming.

The de novo programming changed the constraints of multi-objective problems which initially consisted of several constraints into one constraint in the form of a budget $\sum v_{n} x_{n} \leq B$. Changes into one constraint caused a feasible solution to inequality to be $\sum v_{n} x_{n} \leq B$ an area bounded by lines connected by the following points:

$$
\left(\frac{B}{v_{1}}, 0, \ldots, 0\right) ;\left(0, \frac{B}{v_{2}}, \ldots, 0\right) ; \ldots ;\left(0, \ldots, 0, \frac{B}{v_{n}}\right)
$$

These points are corner points or extreme points of inequality $\sum v_{n} x_{n} \leq B$. In linear programming, the optimum solution of an objective occurs at one of the extreme points of constraint.

Determination of objectives in this study uses the average concept. The average is a measure of data centralization or a measure that describes the centralization of the data set that can represent all data. The average concept can provide a solution to focus decision variables that are different from several objective functions. This data centralization aims to balance the determination of the goal by considering other objectives.

The average concept in setting goals can generally be written as follows:

$$
\bar{z}_{k}=\left[z_{k}\left(x_{1 \operatorname{maxz}_{1}}, \ldots, x_{n \operatorname{maksz}_{1}}\right)+\cdots+z_{k}\left(x_{1 \max _{r}}, \ldots, x_{n \max _{r}}\right)\right] / r
$$


with, $\bar{z}_{k}$ is the objective function goal for- $k$, for $k=$ $1,2, \ldots, r, x_{n m a k s z}$ the ideal variable decision variable is the objective function, $r$ the number of objective functions.

\section{Modification of Goal Programming}

The goal programming is an important technique for making decisions in solving decision-making problems with many goals in finding a satisfactory set of solutions. The goal programming was first introduced by Charnes dan Cooper [4], and further developed by Lee [6], Ignizio [5], Romero [9], Tamiz et. all. [10], Pal [8] and Chang [3]. The goal programmingpurpose to minimize deviations in achieving the goals and levels of the target. The goal programming is generally stated as follows:

$$
\min a=\sum_{k=1}^{r}\left(d_{k}^{+}+d_{k}^{-}\right)+\sum_{l=1}^{s}\left(d_{l}^{+}+d_{l}^{-}\right)
$$

s.t.

$$
\begin{gathered}
f_{k}+d_{k}^{-}-d_{k}^{+}=b_{k}, k=1,2, \ldots, r \\
f_{l}+d_{l}^{-}-d_{l}^{+}=b_{l}, l=1,2, \ldots, s \\
a_{i 1} x_{1}+a_{i 2} x_{2}+\cdots+a_{i n} x_{n} \leq b_{i}, i=1,2, \ldots, m \\
d_{k}^{-}-d_{k}^{+} \geq 0 \\
d_{l}^{-}-d_{l}^{+} \geq 0 \\
x_{j} \geq 0, j=1,2, \ldots, n
\end{gathered}
$$

with (7) is the objective function, (8) maximum objective constraint function, (9) minimum objective constraint function, (10) system constraint, (11) positive and negative deviation for maximum objective, (12) positive and negative deviation for the objective minimum, and (13) non negative constraints.

The de novo programming only has the maximum goal, so the minimum objective function on the goal programming is not needed. In goal programming, the objective function to be achieved is an objective constraint or also called the objective constraint function, so that all the objectives to be achieved in the de novo programming are constraints on the objective function, while the initial constraints of the de novo programming problem are system constraints on the goal programming. Subsequently substituting the problem of the multi-objective de novo programming into the goal programming, as follows:

$$
\min a=\sum_{k=1}^{r} d_{k}^{+}
$$

s.t.

$$
\begin{gathered}
z_{k}-d_{k}^{+}=, k=1,2, \ldots, r \\
a_{i 1} x_{1}+a_{i 2} x_{2}+\cdots+a_{i n} x_{n} \leq b_{i}, i=1,2, \ldots \\
d_{k}^{+} \geq 0 \\
x_{j} \geq 0, j=1,2, \ldots, n
\end{gathered}
$$

with (14) is the objective function, (15) maximum objective function constraints, (16) system constraints, (17) maximum goal deviation, and (18) non negative constraints.

To solve multi-objective problems in the goal programming, the limits of the function constraints of the goals or objectives must be determined in advance. In this study, the goal is to uses inequality (6). So, the modification of the goal programming formula for solving multi-objective de novo programming, as follows:

$$
\min a=\sum_{k=1}^{r} d_{k}^{+}
$$

s.t.

$$
\begin{gathered}
z_{k}-d_{k}^{+}=\bar{z}_{k}, k=1,2, \ldots, r \\
a_{i 1} x_{1}+a_{i 2} x_{2}+\cdots+a_{i n} x_{n} \leq b_{i}, i=1,2, \ldots, m \\
d_{k}^{+} \geq 0 \\
x_{j} \geq 0, j=1,2, \ldots, n
\end{gathered}
$$

with $\bar{z}_{k}$ is the goal for- $k$.

\section{Numerical Examples}

The example refers to the numerical problems of Zeleny [13]

$$
\begin{gathered}
\max z_{1}=50 x_{1}+100 x_{2}+17.5 x_{3} \text { (Profit) } \\
\max z_{2}=92 x_{1}+75 x_{2}+50 x_{3} \text { (Quality) } \\
\max z_{3}=25 x_{1}+100 x_{2}+75 x_{3} \text { (Satisfaction) }
\end{gathered}
$$

s.t.

$$
\begin{gathered}
12 x_{1}+17 x_{2} \leq 1400 \text { (Milling Machine) } \\
3 x_{1}+9 x_{2}+8 x_{3} \leq 1000 \text { (Lathe) } \\
10 x_{1}+13 x_{2}+15 x_{3} \leq 1750 \text { (Burs) } \\
6 x_{1}+16 x_{3} \leq 1325 \text { (Jigsaw) } \\
12 x_{2}+7 x_{3} \leq 900 \text { (Drill) } \\
9.5 x_{1}+9.5 x_{2}+4 x_{3} \leq 1075 \text { (Bad saw) } \\
x_{1}, x_{2}, x_{3} \geq 0
\end{gathered}
$$

with resource unit prices $p_{1}=\$ 0.75, p_{2}=\$ 0.6, p_{3}=$ $0.35, p_{4}=\$ 0.5, p_{5}=\$ 1.15, p_{6}=\$ 0.65$. First, change the goal constraints into the de novo programming using inequality (2), so that it is obtained:

$$
\begin{gathered}
z_{1}=50 x_{1}+100 x_{2}+17.5 x_{3} \\
z_{2}=92 x_{1}+75 x_{2}+50 x_{3} \\
z_{3}=25 x_{1}+100 x_{2}+75 x_{3}
\end{gathered}
$$

s.t. 


$$
\begin{gathered}
23.475 x_{1}+42.675 x_{2}+28.7 x_{3} \leq 4658.75 \\
x_{1}, x_{2}, x_{3} \geq 0 .
\end{gathered}
$$

Then calculate the value of the maximum ideal solution for each objective function against the constraints. The results of calculating the maximum ideal solution are presented in Table 1.

Table 1. Decision Variables and Maximum Ideal Solutions.

\begin{tabular}{llll}
\hline Var. Decisison & $\boldsymbol{z}_{\mathbf{1}}$ & $\boldsymbol{z}_{\mathbf{2}}$ & $\boldsymbol{z}_{\mathbf{3}}$ \\
\hline$x_{1}$ & 0 & 198.456 & 0 \\
$x_{2}$ & 109.168 & 0 & 0 \\
$x_{3}$ & 0 & 0 & 162.326 \\
Sol. Ideal Max & 10916.813 & 18257.934 & 12174.434 \\
\hline
\end{tabular}

Table 1 shows the maximum ideal solution value of the objective function $z_{k}$, namely $z=(10916.813,18257.934$, 12174.434). Next, calculate the value of the objectives to be achieved for each goal using equation (4). The results of calculating the target values for each goal are presented in Table 2.

Table 2. Value of Goals.

\begin{tabular}{ll}
\hline Objective function & Value Objective \\
\hline$\overline{z_{1}}$ & 7893.435 \\
$\overline{z_{2}}$ & 11520.611 \\
$\overline{z_{3}}$ & 9350.666 \\
\hline
\end{tabular}

Table 2 shows the value of the objectives to be achieved for each goal. Next to solve the multi-objective problem the de novo programming uses a goal programming, namely as follows:

$$
\min a=d_{1}^{+}+d_{2}^{+}+d_{3}^{+}
$$

s.t.

$$
\begin{gathered}
50 x_{1}+100 x_{2}+17.5 x_{3}-d_{1}^{+}=7893.435 \\
92 x_{1}+75 x_{2}+50 x_{3}-d_{2}^{+}=11520.611 \\
25 x_{1}+100 x_{2}+75 x_{3}-d_{3}^{+}=9350.881 \\
23.475 x_{1}+42.675 x_{2}+28.7 x_{3} \leq 4658.75 \\
d_{1}^{+}, d_{2}^{+}, d_{3}^{+} \geq 0 \\
x_{1}, x_{2}, x_{3} \geq 0
\end{gathered}
$$

Computational calculations using the help of the excel solver programming. The computational results are presented in Table 3.

Table 3. Decision and Maximum Variable Values Objective Function.

\begin{tabular}{llll}
\hline Var. Decision & $\boldsymbol{z}_{\mathbf{1}}$ & $\boldsymbol{z}_{\mathbf{2}}$ & $\boldsymbol{z}_{\mathbf{3}}$ \\
\hline$x_{1}$ & 66.154 & 66.154 & 66.154 \\
$x_{2}$ & 36.389 & 36.389 & 36.389 \\
$x_{3}$ & 54.106 & 54.106 & 54.106 \\
Maxsimum Value & 7893.435 & 11520.661 & 9350.666 \\
\hline
\end{tabular}

Based on the value of the decision variable obtained, the design of the money system proposed for the constraints limit based on the available budget is presented in Table 4 .
Table 4. Proposed Limit Constraints.

\begin{tabular}{ll}
\hline Machine Type & New Constraints Limits \\
\hline Milling Machine & 1412.461 \\
Lathe & 958.811 \\
Burrs & 1946.187 \\
Jigsaw & 1262.620 \\
Drill & 815.410 \\
Band saw & 1190.583 \\
\hline
\end{tabular}

Table 4 presents proposed new constraints given by the de novo programming based on the available budget, so that the prepared resources can be fully utilized.

Comparison of the previous method is presented in the following table:

Table 5. Comparison of Decision Variables.

\begin{tabular}{llllll}
\hline Var. & Zeleny & Li & Umarusman & Banik & Afli \\
\hline$x_{1}$ & 92.48 & 111.43 & 98.124 & 198.45 & 66.154 \\
$x_{2}$ & 20.90 & 7.60 & 6,69 & 0.0008 & 36.389 \\
$x_{3}$ & 55.61 & 59.89 & 72.116 & 0 & 54.106 \\
\hline
\end{tabular}

Table 6. Comparison of the Maximum Value of the Objective Function.

\begin{tabular}{llll}
\hline Objective Function & Zeleny & Li & Umarusman \\
\hline$z_{1}$ & 7686.87 & 7379.13 & 6837.348 \\
$z_{2}$ & 12855.89 & 13815.46 & 13135.11 \\
$z_{3}$ & 8572.40 & 8037.02 & 8530.93 \\
Objective Function & Banik & Afli & \\
$z_{1}$ & 9922.81 & 7893.435 & \\
$z_{2}$ & 18257.46 & 11520.611 & \\
$z_{3}$ & 4961.43 & 9350.666 & \\
\hline
\end{tabular}

Table 7. Comparison of Proposed Limit Constraints.

\begin{tabular}{llll}
\hline Machine Type & Available & Zeleny & Li \\
\hline Miling Limit & 1400 & 1465.06 & 1466,36 \\
Lathe & 1000 & 910.42 & 881,81 \\
Burrs & 1750 & 2030.65 & 2111,45 \\
Jigsaw & 1325 & 1444.64 & 1626,82 \\
Drill & 900 & 640.07 & 510,430 \\
Bad saw & 1075 & 1299.55 & 1370,35 \\
Machine Type & Umarusman & Banik & Afli \\
Miling Limit & 1291.24 & 2381,46 & 1412,461 \\
Lathe & 931.52 & 595,37 & 958,811 \\
Burrs & 2149.98 & 1984,55 & 1946,187 \\
Jigsaw & 1742.61 & 1190,73 & 1262,620 \\
Drill & 585.104 & 0,010 & 815,410 \\
Bad saw & 1284.21 & 1885,32 & 1190,583 \\
\hline
\end{tabular}

\section{Conclusion}

The multi-objective problem of the de novo programming is a matter of general multi-objective programming whose constraints are constructed into the form of a budget. The de novo programming in addition to providing solutions to multi-objective problems also provides a more optimal system design proposal based on the available budget to increase production yields and maximize the use of raw materials. There are several methods can be uses to solve the multi-objective problem of the de novo programming. In this study the authors uses a modified goal programming approach to solve multi-objective de novo programming.

Modifications made are in determining the objectives of 
each goal. Determination of objectives in this study uses the average concept. The use of the average concept gives the target value for a objective by considers the other objectives, so that the solution obtained is not the most ideal solution for one objective but for all objectives.

The average concept in this study is used for the constraint function which consists of one constrain as a de novoprogramming problem that converts several constraint functions into one constraint function in the form of a budget. This flat concept applies to all objectives that have the same interests, so that the objectives to be achieved are determined using the average concept.

\section{Acknowledgements}

I would like to express my gratitude to my advisor, Dr. M. D. H. Gamal and my mentors for their encouragement, constant support, helpful remarks and insightful comments on the paper.

\section{References}

[1] Z. Babic and I. Pavic, "Multicriterial production programming by program de novo approach," International Journal of Production Economics, vol. 43, pp. 59-66, April 1992.

[2] S. Banik and D. Bathacarya, "Weigthed goal programming approach for solving multi-ojective de novo programming problems," IJERCSE, vol. 5, pp. 316-322, February 2018.

[3] T. Chang, "Multi-choise goal programming,"Omega, vol. 35, pp. 389-396, August 2007.

[4] A. Charnes dan W. W. Cooper, Management Model and Industrial Aplication of Linear Programming, Wiley, New York, 1961.

[5] J. P. Ignizio, Introduction to Linear Goal Programming, Sage, Beverly Hills, 1985.
[6] S. M. Lee, Goal Programming for Decision Analysis, Auerbach, Philadelphia, 1972.

[7] R. J. Li and E. S. Lee, "Fuzzy approaches to multicriteria de novo programmings," Journal of Mathematical Analysis and Aplications, vol. 153, pp. 97-111, July 1989.

[8] B. B. Pal, B. N. Moitra and U. Maulik, "A goal programming procedure for fuzzy multiobjective linear fractional programming problem," Fuzzy Set and Systems, vol. 139, pp. 395-405, October 2003.

[9] C. Romero, "Extended lexicographic goal programminging: A unifying approach," Omega, vol. 29, pp. 63-71, February 2001.

[10] M. Tamiz, D. Jones dan C. Romero, "Goal programming for decision making: An overview of the current state-of-the-art", European Journal of Operational Research, vol. 111, pp. 569581, December 1998.

[11] N. Umarusman, "Min-max goal programming approach for solving multi-objective programming de novo poblems,"IJOR, vol. 10, pp. 92-99, April 2013.

[12] M. Zeleny, "Optimal system design with multiple criteria: Denovo programming approach", Engineering Cost and Production Economics, vol. 10, pp. 89-94, 1986.

[13] M. Zeleny, "Optimizing given system vs. designing optimal system: the de novo programming approach," International Journal of General Systems, vol. 17, pp. 295-307, September 1989.

[14] Y. M. Zhang, G. H. Huang and X. D. Zhang, "Inexact program de novo for water resource systems planing," European Joirnal of Operation Research, vol. 199, pp. 531-541, December 2009.

[15] Z. Y. Zuang and A. Hocine, "Meta goal programming approach for solving multi-objective program de novo problems" European Journal of Operatian Research, vol. 265, pp. 228-238, July 2017. 